\title{
An Analysis of the Temporal Correlation of Interference in Extended Wireless Networks
}

\author{
C. Emre Koksal \\ Department of Electrical and Computer Engineering \\ The Ohio State University Columbus, Ohio 43201
}

\begin{abstract}
We consider an extended wireless network, in which nodes arrive in the network according to a joint spatio-temporal (possibly non-homogenous) Poisson process. We assume that each node remains active for a random duration of time with a certain distribution, independent of the arrival process. During the activity period, each node transmits a signal from a Gaussian alphabet at a fixed power (variance) level. We derive the autocovariance function of the interference experienced by a node located at any given point in the network. We show that, the spatial and the temporal components of the autocovariance function are separable and they take effect in the form of a product. Also, somewhat surprisingly, the "shape" of the autocovariance function is dictated by the service distribution of a single node. Hence, the bandwidth of the cumulative interference is identical to that, caused by a single node. Finally, if duration of the activity period is heavy tailed, then the interference process becomes long range dependent.
\end{abstract}

\section{INTRODUCTION}

Multiuser interference has been a significant determinant of the performance in wireless communication. When a message is transmitted over a block of channel uses, large enough to invoke random coding arguments, to determine the success of a transmission, the accumulated mutual information is compared with the encoding rate. In the Gaussian channel, this reduces to the comparison of the observed signal to interference and noise ratio (SINR) with a threshold, which is a function of the encoding rate. If the SINR remains below the threshold, an outage occurs and the block cannot be decoded with an arbitrarily low probability of error.

To calculate the probability of outage, for a given signal and noise power, it is typical to use the sample distribution of the interference process. The inherent assumption here is that, the interference remains constant over the duration of the block of interest. However, with many of the new generation codes (e.g., rateless codes, incremental redundancy codes, etc.), sizes of the blocks across which the information is encoded can be fairly large. Consequently, the interference level may vary significantly across the block and sample statistics of the interference process is not sufficient to analyze the outage probability. There is a need to study of temporal statistics of the interference in order to analyze the outage probability and to design efficient codes to mitigate the effects of the interference.

To that end, we study the following setup. We consider an extended wireless network in which nodes arrive according to a joint spatio-temporal (possibly non-homogenous) Poisson process. We assume that each node remains active for a random duration of time with a certain distribution, independent of the arrival process. During the activity period, each node transmits a signal from a Gaussian alphabet at a fixed power level. Thus, the number of active nodes can be modeled as the number of customers in and $\mathrm{M} / \mathrm{G} / \infty$ queue. We derive the autocovariance function of the interference experienced by a node located at any given point in the network. Toward that goal, we first find the autocovariance function for the number of customers in an $\mathrm{M} / \mathrm{G} / \infty$ queue and use this to derive the autocovariance function for the interference. This derivation will provide the main insights and will lead to the subsequent derivation of the autocovariance function of the interference.

The main observations we deduce from the analysis are the following: As long as the intensity function of the arrival process is not dependent on the observed interference or the service duration,

(1) The spatial and the temporal components of the autocovariance function are separable and they take effect in the form of a product.

(2) The "shape" of the autocovariance function is dictated by the service distribution of a single node. Hence, the bandwidth of the cumulative interference is identical to that, caused by a single node.

(3) If duration of the activity period (e.g., the size of the file to be transmitted) is heavy tailed, then the interference process becomes long range dependent.

The dominance of single node statistics in the cumulative interference is somewhat surprising, since, as the network size extends, the number of simultaneous interferers approaches $\infty$. One might think that the total interference becomes whiter and whiter, as the number of interferers increase, however this does not turn out to be true. The node arrival intensity and the transmission power affect the scale of the covariance function, but not the shape of it. In a sense, the temporal behavior of the interference is the same regardless of whether there is a single interferer or many interferers.

Note that our model for the node arrival and service process is suitable for the scenarios in which node arrival dynamics vary in smaller time scales, e.g., in networks where the arriving nodes, transmit very small amount of information and depart. With applications requiring such intermittent and delay-sensitive data requests with short-lived connections, congestion avoidence schemes start to become more inefficient and the approach of all nodes transmitting upon arrival may become more appealing [1].

Other studies on temporal modeling of the interference include [2], [3]. The techniques introduced in our paper to 
analyze interference are based on queuing theory and are significantly different from these papers, leading to various new insights. In [4], [5], [6], [7], the temporal correlation analyses focus on a particular interference avoidence strategies that are variants of ALOHA. In our model, we assume that there is no collision avoidance or scheduling, which is appropriate for a completely different set of MAC schemes, including direct sequence CDMA.

\section{System Model and Problem Statement}

We consider an extended wireless network, in which nodes arrive according to a joint spatio-temporal (possibly nonhomogenous) Poisson process. In particular, the number of nodes that become active within any given duration of time is a Poisson random variable and at any point in time, the spatial distribution of the active nodes form a 2D Poisson process. We denote the intensity of this process by $\lambda(t, x, y)$ nodes $/ \mathrm{sec} / \mathrm{m}^{2}$, which is the arrival rate of nodes at location $(x, y)$ (represented in Cartesian coordinates) at time $t$. The period over which a node remains active is i.i.d. over each user and is represented with a random variable $T$ that has a distribution $F(t)$. A node emits power during the entirety of its activity period, i.e., we assume no collision avoidance or scheduling scheme to be in effect.

We assume a fixed-power random Gaussian signalling by each node during its entire activity period and denote symbol transmitted at time $t$ by a node that becomes active in $((i-1) \delta, i \delta)$ at location $(x, y)$ with $\sqrt{P} W_{i}(t, x, y)$, where $W_{i}(t, x, y) \sim \mathcal{C N}\left(\mathbf{0}, \frac{1}{\sqrt{2}} I\right)$ and $P$ denotes the transmission power. The instantaneous channel gain between points $(x, y)$ and $\left(x^{\prime}, y^{\prime}\right)$ is given by $G\left(x, y, x^{\prime}, y^{\prime}, t\right)$ and the associated power gain is $H\left(x, y, x^{\prime}, y^{\prime}, t\right) \triangleq\left|G\left(x, y, x^{\prime}, y^{\prime}, t\right)\right|^{2}$. We assume channel reciprocity: $G\left(x, y, x^{\prime}, y^{\prime}, t\right)=G\left(x^{\prime}, y^{\prime}, x, y, t\right)$, for simplicity. Also, we assume $G\left(x, y, x^{\prime}, y^{\prime}, t\right)$ to be continuous in both temporal and spatial parameters. In other words, we assume the variations in the electric field intensity is not abrupt. Indeed, typically, significant variations occur at spatial distances of order, comparable to the wavelengths, even in rich scattering environments.

We define the interference level observed at location $(x, y)$ at time $t$ as $Z(t, x, y)$. We assume $\lambda(t, x, y)$ and $G\left(x, y, x^{\prime}, y^{\prime}, t\right)$ to be exogenous processes. Note that the user intensity, $\lambda(t, x, y)$, is not influenced by the interference level $Z(t, x, y)$ in the network. This is in contrast to a carriersense based MAC scheme, where the intensity is influenced by the interference measured (via sensing of the carrier). In this paper, we also assume that $\lambda(t, x, y)$ is independent of $G\left(x, y, x^{\prime}, y^{\prime}, t\right)$ for all $(x, y)$. However, we also provide the generalization of the result for the scenario in which $\lambda(t, x, y)$ depends $^{1}$ on the instantaneous channel gain $H\left(x, y, x^{\prime}, y^{\prime}, t\right)$.

Finally, we define the indicator variable

\footnotetext{
${ }^{1}$ The dependency of $\lambda(t, x, y)$ and $G\left(x, y, x^{\prime}, y^{\prime}, t\right)$ implies that a node activation policy is be in effect, that influences the node arrivals depending on only the gain of the channel over which the node is going to transmit. This admission control policy accepts or drops the nodes as a function of an exogenous channel gain process, $G\left(x, y, x^{\prime}, y^{\prime}, t\right)$.
}

$I_{i}(x, y, t) \triangleq \begin{cases}1, & \text { a node at location }(x, y) \text { becomes active in } \\ & ((i-1) \delta, i \delta) \text { and is still active at time } t \\ 0, & \text { otherwise }\end{cases}$

We can write the following for the observed interference at point $(x, y)$ :

$$
\begin{aligned}
& Z(t, x, y)=\sum_{i=-\infty}^{t / \delta} \sum_{\kappa=-\infty}^{\infty} \sum_{\gamma=-\infty}^{\infty} \\
& \underbrace{\sqrt{P} I_{i}(\gamma \Delta, \kappa \Delta, t) G(x, y, \gamma \Delta, \kappa \Delta, t) W_{i}(t, \gamma \Delta, \kappa \Delta)}_{\triangleq Z_{i, \kappa, \gamma}(t, x, y)} .
\end{aligned}
$$

Here, one can realize that the temporal and spatial increments are in steps of $\delta$ seconds and $\Delta$ meters, respectively. One can view $\delta$ as the transmission time of a symbol (roughly identical to the reciprocal of the system bandwidth) and $\Delta$ as the distance covered by half the wavelength associated with the center frequency. With the zero-mean Gaussian sampling, $\mathbb{E}[Z(t, x, y)]=\mathbf{0}$. For simplicity, in the sequel, we will use $\sum_{i, \kappa, \gamma}$ to denote the triple summation $\sum_{i=-\infty}^{t / \delta} \sum_{\kappa=-\infty}^{\infty} \sum_{\gamma=-\infty}^{\infty}$.

Note that interference process $\{Z(t, x, y)\}$ is uncorrelated in time for any location $(x, y)$, since it is driven by a combination of zero-mean i.i.d. processes ${ }^{2}\left\{W_{i}\left(t, x^{\prime}, y^{\prime}\right), t \geq-\infty\right\}$ for all locations $\left(x^{\prime}, y^{\prime}\right)$.

In this paper, we evaluate the temporal covariance function, $K_{Z_{\mathcal{P}}(x, y)}(t, \tau)$, of the instantaneous power, $Z_{\mathcal{P}}(t, x, y) \triangleq$ $|Z(t, x, y)|^{2}$ of the interference. Thus,

$$
Z_{\mathcal{P}}(t, x, y)=\sum_{i, \kappa, \gamma} \sum_{i^{\prime}, \kappa^{\prime}, \gamma^{\prime}} Z_{i, \kappa, \gamma}(t, x, y) \cdot Z_{i^{\prime}, \kappa^{\prime}, \gamma^{\prime}}^{*}(t, x, y)
$$

\section{Autocovariance Function of the Number of ACTIVE Nodes PER UNIT AREA}

In our model, the number of nodes per any unit area of the network follows an $\mathrm{M} / \mathrm{G} / \infty$ queue. In our derivation, we first rederive the autocovariance function for the number of customers in an $\mathrm{M} / \mathrm{G} / \infty$ queue, which was first found in [8]. There, Cox represented the number of nodes in a differential form. Here, we rederive the same result using special indicator variables. This derivation will provide the main insights and will lead to the subsequent derivation of the autocovariance function of the interference.

Let the $\mathrm{M} / \mathrm{G} / \infty$ queue be operating since time $-\infty$ and $N(t)$ be the number of active nodes in a given unit area region at time $t$. Let the node arrival rate be $\lambda$ nodes/sec for that unitarea region (a homogenous Poisson process for simplicity in this section). Since, we are merely concerned with the number of nodes, the indicator variable defined in (1) can be simplified to:

\footnotetext{
${ }^{2}$ Note to get some intuition on this, consider a Markov-modulated process, $\{X[n], n>-\infty\}$ with two states, ON and OFF. In the ON state, the values of $X[n]$ are picked from an i.i.d. process $W[n]$ with a finite first moment $\bar{W}$, and in the OFF state, $X[n]=0$. Let $S[n]$ represent the state at time $n$. Process $\{X[n]\}$ is stationary with an autocovariance function, $K_{X X}[n, m]=\bar{W}^{2}\left[\mathbb{P}(S[n]=S[m]=1)-\mathbb{P}^{2}(S[n]=1)\right]$. Thus, even though $X[n]$ and $X[\mathrm{~m}]$ are statistically dependent, they are uncorrelated if $\bar{W}=0$ and correlated otherwise.
} 
$I_{i}(t) \triangleq\left\{\begin{array}{cc}1 & \begin{array}{c}\text { a node arrives in }((i-1) \delta, i \delta) \text { and still in } \\ \text { the unit-area region at time } t\end{array} \\ 0 & \text { otherwise }\end{array}\right.$.

for $\delta$ being the amount of temporal increment. Therefore,

$$
N(t)=\sum_{i=-\infty}^{t / \delta} I_{i}(t)
$$

For any $t, \tau$ such that $t \leqslant \tau$,

$$
\begin{aligned}
& \mathbb{E}[N(t) N(\tau)]=\mathbb{E}\left[\sum_{i=-\infty}^{t / \delta} \sum_{j=-\infty}^{\tau / \delta} I_{i}(t) I_{j}(\tau)\right] \\
& =\sum_{\{i \leqslant t / \delta, j \leqslant \tau / \delta \mid} \mathbb{E}\left[I_{i}(t) I_{j}(\tau)\right]+\sum_{i=-\infty}^{t / \delta} \mathbb{E}\left[I_{i}(t) I_{i}(\tau)\right] \\
& =\sum_{\{i \leqslant t / \delta, j \leqslant \tau / \delta \mid i \neq j\}} \mathbb{E}\left[I_{i}(t)\right] \mathbb{E}\left[I_{j}(\tau)\right]+\sum_{i=-\infty}^{t / \delta} \mathbb{E}\left[I_{i}(t) I_{i}(\tau)\right],
\end{aligned}
$$

where Eq. (5) follows since $I_{i}(t)$ and $I_{j}(\tau)$ are independent for $i \neq j$. We can also write

$$
\begin{array}{r}
\mathbb{E}[N(t)] \mathbb{E}[N(\tau)]=\mathbb{E}\left[\sum_{i=-\infty}^{t / \delta} I_{i}(t)\right] \mathbb{E}\left[\sum_{j=-\infty}^{\tau / \delta} I_{j}(\tau)\right] \\
=\sum_{\{i \leqslant t / \delta, j \leqslant \tau / \delta} \mathbb{E}\left[I_{i}(t)\right] \mathbb{E}\left[I_{j}(\tau)\right] \\
+\sum_{i=-\infty}^{t / \delta} \mathbb{E}\left[I_{i}(t)\right] \mathbb{E}\left[I_{i}(\tau)\right] .
\end{array}
$$

Combining Eq. (5) and (6) we get,

$$
\begin{gathered}
\operatorname{cov}(N(t), N(\tau))=\mathbb{E}[N(t) N(\tau)]-\mathbb{E}[N(t)] \mathbb{E}[N(\tau)] \\
=\sum_{i=-\infty}^{t / \delta}\left\{\mathbb{E}\left[I_{i}(t) I_{i}(\tau)\right]-\mathbb{E}\left[I_{i}(t)\right] \mathbb{E}\left[I_{i}(\tau)\right]\right\}
\end{gathered}
$$

One can observe that, since $\tau>t, \mathbb{E}\left[I_{i}(t) I_{i}(\tau)\right]=\mathbb{E}\left[I_{i}(\tau)\right]$. With this observation, one can write

$$
\mathbb{E}\left[I_{i}(t) I_{i}(\tau)\right]-\mathbb{E}\left[I_{i}(t)\right] \mathbb{E}\left[I_{i}(\tau)\right]=\mathbb{E}\left[I_{i}(\tau)\right]\left(1-\mathbb{E}\left[I_{i}(t)\right]\right),
$$

and the following inequalities are immediate:

$$
\begin{aligned}
& \mathbb{E}\left[I_{i}(\tau)\right]\left(1-\mathbb{E}\left[I_{i}(\tau)\right]\right) \leq \\
& \mathbb{E}\left[I_{i}(\tau)\right]\left(1-\mathbb{E}\left[I_{i}(t)\right]\right) \leq \mathbb{E}\left[I_{i}(\tau)\right],
\end{aligned}
$$

where the lower bound follows since $I_{i}(\tau) \leq I_{i}(t)$ with probability 1 . Next, we focus on $\mathbb{E}\left[I_{i}(\tau)\right]$. Using $F^{k}(\cdot) \triangleq[F(\cdot)]^{k}$ for simplicity, we can write:

$$
\begin{aligned}
\mathbb{E}\left[I_{i}(\tau)\right] & =\sum_{k=1}^{\infty}\{\mathbb{P}(k \text { node arrivals in }((i-1) \delta, i \delta)) \\
& =\sum_{k=1}^{\infty}\left\{\frac{e^{-\lambda \delta}(\lambda \delta)^{k}}{k !}\left[1-F^{k}(\tau-i \delta)\right]\right\} \\
& \leq \sum_{k=1}^{\infty}\left\{e^{-\lambda \delta}(\lambda \delta)^{k}\left[1-F^{k}(\tau-i \delta)\right]\right\} \\
& =e^{-\lambda \delta}\left[\sum_{k=1}^{\infty}(\lambda \delta)^{k}-\sum_{k=1}^{\infty}(\lambda \delta F(\tau-i \delta))^{k}\right] \\
& =e^{-\lambda \delta}\left[\frac{\lambda \delta}{1-\lambda \delta}-\frac{\lambda \delta F(\tau-i \delta)}{1-\lambda \delta F(\tau-i \delta)}\right] \\
& =e^{-\lambda \delta} \lambda \delta\left[\frac{1-F(\tau-i \delta)}{(1-\lambda \delta)(1-\lambda \delta F(\tau-i \delta))}\right] \\
& \leq \frac{e^{-\lambda \delta}}{(1-\lambda \delta)^{2}} \cdot \lambda \delta[1-F(\tau-i \delta)],
\end{aligned}
$$

where (9) follows from total probability, (10) follows since the node arrivals are Poisson, and (11) follows since $1-\lambda \delta \leq$ $1-\lambda \delta F(\tau-i \delta)$. Along the same lines, we can also derive the following lower bound:

$$
\begin{aligned}
\mathbb{E}\left[I_{i}(\tau)\right] \geq \mathbb{P}(1 \text { single node arrival in }((i-1) \delta, i \delta)) \\
\cdot[1-F(\tau-i \delta)] \\
=e^{-\lambda \delta} \cdot \lambda \delta[1-F(\tau-i \delta)],
\end{aligned}
$$

where (12) follows since the sum in (9) is lower bounded by the first term of the summation there.

From the upper bound in (8), we have:

$$
\begin{aligned}
\operatorname{cov}(N(t), N(\tau)) & \leq \sum_{i=-\infty}^{t / \delta} \mathbb{E}\left[I_{i}(\tau)\right] \\
& \leq \frac{e^{-\lambda \delta}}{(1-\lambda \delta)^{2}} \sum_{i=-\infty}^{t / \delta} \lambda \delta[1-F(\tau-i \delta)],
\end{aligned}
$$

which follows from (11). Therefore, as $\delta \rightarrow 0^{+}$,

$$
\begin{aligned}
& \operatorname{cov}(N(t), N(\tau)) \leq \int_{-\infty}^{t} \lambda {[1-F(\tau-s)] d s } \\
&=\lambda \int_{|\tau-t|}^{\infty}[1-F(s)] d s .
\end{aligned}
$$

Similarly, from the lower bound in (8), we can write:

$$
\begin{aligned}
& \operatorname{cov}(N(t), N(\tau)) \geq \sum_{i=-\infty}^{t / \delta}\left\{\mathbb{E}\left[I_{i}(\tau)\right]\left(1-\mathbb{E}\left[I_{i}(\tau)\right]\right)\right\} \\
& \geq \sum_{i=-\infty}^{t / \delta}\left\{\mathbb{E}\left[I_{i}(\tau)\right]\left(1-\frac{e^{-\lambda \delta}}{(1-\lambda \delta)^{2}} \cdot \lambda \delta[1-F(\tau-i \delta)]\right)\right\} \\
& \geq \sum_{i=-\infty}^{t / \delta}\left\{\mathbb{E}\left[I_{i}(\tau)\right]\left(1-\frac{e^{-\lambda \delta}}{(1-\lambda \delta)^{2}} \cdot \lambda \delta\right)\right\}
\end{aligned}
$$




$$
\geq e^{-\lambda \delta}\left(1-\frac{e^{-\lambda \delta}}{(1-\lambda \delta)^{2}} \cdot \lambda \delta\right) \sum_{i=-\infty}^{t / \delta} \lambda \delta[1-F(\tau-i \delta)]
$$

where (15) follows from (11); (16) follows since $1-F(\tau-$ $i \delta) \leq 1$, and (17) follows from (12). Hence, similar to (14), as $\delta \rightarrow 0^{+}$,

$$
\operatorname{cov}(N(t), N(\tau)) \geq \lambda \int_{|\tau-t|}^{\infty}[1-F(s)] d s .
$$

Consequently, combining (14) and (18), we conclude:

$$
\operatorname{cov}(N(t), N(\tau))=\lambda \int_{|\tau-t|}^{\infty}[1-F(s)] d s .
$$

One can see that, since the above covariance is a function of the time difference $t-\tau$ only, $\{N(t)\}$ is a stationary process.

\section{Autocovariance Function of the Interference POWER}

Next, we expand the analysis to calculate the autocovariance function of the total combined interference observed by a given node. The derivation follows identical steps, however, we have to be more careful now in keeping track of the distance of the interferers from the origin.

Theorem 1: The interference power process is stationary and its autocovariance function can be written as

$$
\begin{gathered}
K_{Z_{\mathcal{P}}(x, y)}(t-\tau)=P^{2} \int_{-\infty}^{t}[1-F(\tau-s)] \int_{-\infty}^{\infty} \int_{-\infty}^{\infty} \\
\mathbb{E}\left[\lambda\left(s, x^{\prime}, y^{\prime}\right)\right] \mathbb{E}\left[H\left(x, y, x^{\prime}, y^{\prime}, t\right) H\left(x, y, x^{\prime}, y^{\prime}, \tau\right)\right] d x^{\prime} d y^{\prime} d s .
\end{gathered}
$$

Proof: The autocovariance function for the interference power process $\left\{Z_{\mathcal{P}}(t, x, y)\right\}$ can be written as follows:

$$
\begin{gathered}
K_{Z_{\mathcal{P}}(x, y)}(t, \tau)=\operatorname{cov}\left(Z_{\mathcal{P}}(t, x, y), Z_{\mathcal{P}}(\tau, x, y)\right) \\
=\sum_{i, \kappa, \gamma} \sum_{i^{\prime}, \kappa^{\prime}, \gamma^{\prime}}\left\{\mathbb { E } \left[Z_{i, \kappa, \gamma}(t, x, y) Z_{i^{\prime}, \kappa^{\prime}, \gamma^{\prime}}^{*}(t, x, y)\right.\right. \\
\left.\cdot Z_{i, \kappa, \gamma}(\tau, x, y) Z_{i^{\prime}, \kappa^{\prime}, \gamma^{\prime}}^{*}(\tau, x, y)\right] \\
-\mathbb{E}\left[Z_{i, \kappa, \gamma}(t, x, y) Z_{i^{\prime}, \kappa^{\prime}, \gamma^{\prime}}^{*}(t, x, y)\right] \\
\left.\cdot \mathbb{E}\left[Z_{i, \kappa, \gamma}(\tau, x, y) Z_{i^{\prime}, \kappa^{\prime}, \gamma^{\prime}}^{*}(\tau, x, y)\right]\right\}
\end{gathered}
$$

where the expectations are over the joint distribution of channel gains and the node arrival process (as well as the distribution of the node intensity process, if it is random). With a similar approach to the $\mathrm{M} / \mathrm{G} / \infty$ derivation (19), we can break the summation into two parts, one for the terms $i=i^{\prime}, \gamma=\gamma^{\prime}, \kappa=\kappa^{\prime}$ and the other for the rest of the terms. Since all terms for $i \neq i^{\prime}, \gamma \neq \gamma^{\prime}, \kappa \neq \kappa^{\prime}$ are 0 $\left(W_{i}(\cdot, \gamma \Delta, \kappa \Delta)\right.$ is i.i.d. and 0-mean), we have:

$$
\begin{gathered}
K_{Z_{\mathcal{P}}(x, y)}(t, \tau)=\sum_{i, \kappa, \gamma}\left\{\mathbb{E}\left[\left|Z_{i, \kappa, \gamma}(t, x, y)\right|^{2} \cdot\left|Z_{i, \kappa, \gamma}(\tau, x, y)\right|^{2}\right]\right. \\
\left.-\mathbb{E}\left[\left|Z_{i, \kappa, \gamma}(t, x, y)\right|^{2}\right] \cdot \mathbb{E}\left[\left|Z_{i, \kappa, \gamma}(\tau, x, y)\right|^{2}\right]\right\} \\
=\sum_{i, \kappa, \gamma}\left\{\mathbb { E } \left[I_{i}(\gamma \Delta, \kappa \Delta, t) I_{i}(\gamma \Delta, \kappa \Delta, \tau)\right.\right. \\
\quad H(r, \phi, \gamma \Delta, \kappa \Delta, t) H(r, \phi, \gamma \Delta, \kappa \Delta, \tau)] \\
\cdot \mathbb{E}\left[\left|W_{i}(t, \gamma \Delta, \kappa \Delta)\right|^{2}\right] \mathbb{E}\left[\left|W_{i}(\tau, \gamma \Delta, \kappa \Delta)\right|^{2}\right]- \\
\left.\left(\mathbb{E}\left[I_{i}(\gamma \Delta, \kappa \Delta, t) H(r, \phi, \gamma \Delta, \kappa \Delta, t)\right] \mathbb{E}\left[\left|W_{i}(t, \gamma \Delta, \kappa \Delta)\right|^{2}\right]\right)^{2}\right\} \\
=P^{2} \sum_{i, \kappa, \gamma}\left\{\mathbb{E}\left[I_{i}(\gamma \Delta, \kappa \Delta, t) I_{i}(\gamma \Delta, \kappa \Delta, \tau)\right]\right. \\
\cdot \mathbb{E}[H(r, \phi, \gamma \Delta, \kappa \Delta, t) H(r, \phi, \gamma \Delta, \kappa \Delta, \tau)] \\
\left.-\left(\mathbb{E}\left[I_{i}(\gamma \Delta, \kappa \Delta, t)\right] \mathbb{E}[H(r, \phi, \gamma \Delta, \kappa \Delta, t)]\right)^{2}\right\},
\end{gathered}
$$

where (24) follows since $W_{i}(t, \gamma \Delta, \kappa \Delta)$ has unit variance and $\lambda(t, x, y)$ is independent of $G\left(x, y, x^{\prime}, y^{\prime}, t\right)$. Noting that $\mathbb{E}\left[I_{i}(\gamma \Delta, \kappa \Delta, t)\right]=\mathbb{E}[\lambda(t, x, y)] \delta[1-F(t-i \delta)] \Delta^{2}$, with the additional factor of $\Delta^{2}$ for the specific location that the node is arriving at. Similar to (19), we obtain the desired result as $\delta, \Delta \rightarrow 0^{+}$:

$$
\begin{aligned}
& K_{Z_{\mathcal{P}}(x, y)}(t, \tau)=P^{2} \int_{-\infty}^{t}[1-F(\tau-s)] \int_{-\infty}^{\infty} \int_{-\infty}^{\infty} \mathbb{E}\left[\lambda\left(s, x^{\prime}, y^{\prime}\right)\right] \\
& \mathbb{E}\left[H\left(x, y, x^{\prime}, y^{\prime}, t\right) H\left(x, y, x^{\prime}, y^{\prime}, \tau\right)\right] d x^{\prime} d y^{\prime} d s
\end{aligned}
$$

Note that the remaining terms decay in the limit due to the expectation squared in (24) as well as the assumption that the probability of two separate nodes becoming active simultaneously at the same location ${ }^{3}$ in the network is $\mathrm{o}\left(\delta \Delta^{2}\right)$. Note that the above is written for $t \leq \tau$, but the same relationship holds for $t>\tau$ as well. Also, the autocovariance function is a function of $t-\tau$ and thus the interference power process is stationary: $K_{Z_{\mathcal{P}}(x, y)}(t, \tau)=K_{Z_{\mathcal{P}}(x, y)}(t-\tau)$.

Theorem 1 demonstrates that the user arrival rate, transmission power, and the frequency reuse region size affect the scale of the covariance function, but not the shape of it. In a sense, the temporal "behavior" of the interference is the same regardless of whether there is a single interferer or many interferers. To finalize the discussion we note that, if the intensity process $\lambda\left(s, x^{\prime}, y^{\prime}\right)$ depended on the instantaneous channel gain $H\left(x, y, x^{\prime}, y^{\prime}, s\right)$, Theorem 1 would still hold with the integrand in Eq. 20 changed into the following:

$$
\begin{aligned}
& \mathbb{E}\left[\mathbb{E}\left[\lambda\left(s, x^{\prime}, y^{\prime}\right) \mid H\left(x, y, x^{\prime}, y^{\prime}, s\right)\right]\right. \\
& \left.\cdot \mathbb{E}\left[H\left(x, y, x^{\prime}, y^{\prime}, t\right) H\left(x, y, x^{\prime}, y^{\prime}, \tau\right) \mid H\left(x, y, x^{\prime}, y^{\prime}, s\right)\right]\right],
\end{aligned}
$$

where the outer expectation is over the distribution of the instantaneous power gain $H\left(x, y, x^{\prime}, y^{\prime}, s\right)$. This holds since the underlying node arrival process is still a non-homogenous Poisson process, since $G\left(x, y, x^{\prime}, y^{\prime}, t\right)$ is an exogenous process and $\lambda(\cdot) \leftrightarrow G(\cdot) \leftrightarrow Z(\cdot)$ forms a Markov chain.

\footnotetext{
${ }^{3} \mathrm{Here}$, we assume that the symbol duration is much smaller than the activity period and the user density is sufficiently small that the all active nodes are separated by more than a half wavelength of each other.
} 


\section{A. Static Channels V. EXAMPLES}

First, we assume direct line of sight between any two nodes without any multipath effects. We assume a path-loss model with a path loss exponent represented with parameter $\alpha \geq 2$. Without loss of generality, we focus on the interference at the origin (i.e., any given node) and to rule out the near field effect, we assume that the interferers are located at a distance at least $d$ meters away from the origin. Note that, $H(0,0, \gamma \Delta, \kappa \Delta, t)=\left(\Delta \sqrt{\gamma^{2}+\kappa^{2}}\right)^{-\alpha}$ for all $t$ and $\Delta, \kappa$, such that the distance, $\Delta \sqrt{\gamma^{2}+\kappa^{2}}>d$. For a homogenous node arrival process, i.e., $\mathbb{E}[\lambda(t, x, y)]=\lambda$, converting the integral in (20) into polar coordinates, we obtain:

$$
\begin{gathered}
K_{Z_{\mathcal{P}}(x, y)}(t-\tau)=P^{2} \int_{-\infty}^{t}[1-F(\tau-s)] \\
\int_{-\pi}^{\pi} \int_{0}^{\infty} \lambda\left(d+r^{\prime}\right)^{-2 \alpha+1} d r^{\prime} d \phi^{\prime} d s \\
=\frac{\pi \lambda P^{2}}{\alpha-1} d^{2-2 \alpha} \cdot \int_{|\tau-t|}^{\infty}[1-F(s)] d s .
\end{gathered}
$$

where $r^{\prime}=\sqrt{x^{2}+y^{2}}$ and $\phi^{\prime}=\tan ^{-1}(y / x)$. Here, one can view the "polar equivalent" of the node intensity as $\lambda(t, r, \phi) \triangleq \lambda r$.

One can observe that, the autocovariance function separates into two factors, one of them involving only the temporal components and the other one involving only the spatial components. Note that, following (27),

$$
\begin{aligned}
& \mu_{Z_{\mathcal{P}}}=\mathbb{E}\left[Z_{\mathcal{P}}(t, x, y)\right]=\frac{2 \pi \rho P}{\alpha-2} d^{2-\alpha} \text { and } \\
& \sigma_{Z_{\mathcal{P}}}^{2}=\operatorname{var}\left(Z_{\mathcal{P}}(t, x, y)\right)=\frac{\pi \rho P^{2}}{\alpha-1} d^{2-2 \alpha},
\end{aligned}
$$

where $\rho \triangleq \lambda \mathbb{E}$ [duration for a node remaining active] is the utilization factor (per unit area) for the network.

\section{B. I.I.D. Channels}

In the second scenario, we assume no line of sight. Each channel between any pair of nodes is dominated by multipath fading. Let the power gain $H\left(x, y, x^{\prime}, y^{\prime}, t\right)$ be i.i.d. in time and space with a mean $\bar{H}$ within a radius of $d$, and the power gain (and hence the interference) outside that radius is negligible. This model is more appropriate for an indoor/rich scattering setting. Then, similar to the previous derivation, under polar coordinates with $\lambda(s, x, y)=\lambda$,

$$
\begin{aligned}
K_{Z_{\mathcal{P}}(x, y)}(t-\tau) & =P^{2} \int_{-\infty}^{t}[1-F(\tau-s)] \\
& \int_{-\pi}^{\pi} \int_{0}^{\infty} \lambda r^{\prime} d r^{\prime} d \phi^{\prime} d s \\
& =\pi d^{2} \bar{H}^{2} P^{2} \lambda \int_{|\tau-t|}^{\infty}[1-F(s)] d s
\end{aligned}
$$

One can realize from (27) and (30) that, the autocovariance function depends on the distribution of duration $T$ of active transmission for a single user. Another major conclusion is that, if $T$ is heavy tailed, then the autocovariance functions as given in (27) and (30) decays slower than $|t-\tau|^{-2}$ leading to a long range dependent interference process.

\section{CONClusions}

We derived the autocovariance function of the interference power experienced at a random location in an extended wireless network. We assumed nodes arrive according to a non-homogenous spatio-temporal Poisson process; each node remains active for a random duration of time, independently of the arrival process; and each node transmits a signal from a Gaussian alphabet at any fixed power level.

Our findings have several implications. As the network size extends, since the number of simultaneous interferers approach to $\infty$, one might think that the interference becomes whiter. We showed that this is not true and the covariance function for the interference in the extended network is determined by the service duration of a single node. In a sense, the temporal second order statistics of the interference is the same regardless of whether there is a single interferer or many interferers. An artifact of our analysis was the realization that, if the activity duration is heavy tailed, the interference process is long range dependent regardless of the size or the density, $\lambda$, of the network. A subsequent future question to focus on may be how to design receivers that act satisfactory under colored interference.

\section{REFERENCES}

[1] N. Sugavanam, C. E. Koksal, and A. Eryilmaz, "Color of interference and joint encoding and medium access in large wireless networks," in Proceedings of the Asilomar Conference on Signals, Systems, and Computers, 2012, pp. 1189-1193.

[2] X. Yang and A. Petropulu, "Co-channel interference modeling and analysis in a poisson field of interferers in wireless communications," Trans. Sig. Proc., vol. 51, no. 1, pp. 64-76, Jan. 2003.

[3] K. Gulati, B. L. Evans, and S. Srikanteswara, "Joint temporal statistics of interference in decentralized wireless networks." Trans. Sig. Proc., vol. 60, no. 12 , pp. 6713-6718, 2012, to appear.

[4] M. Haenggi and R. Smarandache, "Diversity Polynomials for the Analysis of Temporal Correlations in Wireless Networks," http://www.arxiv.org/abs/1306.1556.

[5] R. K. Ganti and M. Haenggi, "Spatial and temporal correlation of the interference in aloha ad hoc networks," Comm. Letters., vol. 13, no. 9 pp. 631-633, Sep. 2009.

[6] M. Haenggi and R. K. Ganti, Interference in Large Wireless Networks, ser. Foundations and Trends in Networking. Hanover, MA: now Publishers, Inc., 2008.

[7] J. G. Andrews, R. K. Ganti, M. Haenggi, N. Jindal, and S. Weber, "A primer on spatial modeling and analysis in wireless networks," IEEE Communications Magazine, vol. 48, no. 11, Nov. 2010.

[8] D. R. Cox, Renewal Theory. London, UK: Methuen \& co., Ltd., 1967. 